\title{
Green's function in partial subdivision networks
}

\author{
Ángeles Carmona, Margarida Mitjana, Enric Monsó \\ Departament de Matemàtiques \\ Universitat Politècnica de Catalunya. Spain
}

\begin{abstract}
In the present work, we define a partial subdivision network $\Gamma^{S}$ of a given network $\Gamma$, by inserting a new vertex in some selected edges of $\Gamma$, so that each of these edges is replaced by two new edges with conductances that fulfill the Kirchhoff series law on the new network. Then, we obtain an expression for the Green kernel of the partial subdivision network in terms of the Green kernel of the base network. For that, we show the relation between Poisson problems on the partial subdivision network and Poisson problems on the base network. Moreover, we also obtain the effective resistance and the Kirchhoff index of the partial subdivision network in terms of the corresponding parameters on the base network. Finally, as an example, we carry out the computations in the case of a star network in which we have subdivided the even edges.
\end{abstract}

Keywords: Resistance distance, Green kernel, Kirchhoff Index, partial subdivision network MSC: 31C20, 15A09, 34B45

\section{Introduction}

The subdivision graph of a given graph is obtained by dividing each edge into two edges by inserting one new node. This operation is sometimes called barycentric subdivision of the graph and is important when studying homeomorphic graphs; see [12]. In the literature, we can find some works studying parameters such as effective resistances, Kirchhoff Index, or spectra of subdivision graphs; see $[4,7,9,11,13,15]$. In [8], balanced subdivision graph was studied in order to optimize the largest eigenvalue of the adjacency matrix. The subdivision of graphs is also used to construct equiarboreal graphs in [16]. The authors in [6], carried out the study of subdivision of networks. Specifically, we obtained the expression of the group inverse of the Laplacian matrix of the subdivision network in terms of the group inverse of the Laplacian matrix of the initial network.

In the present paper, we first introduce the concept of partial subdivision of a network, that generalizes the usual subdivision network, in the sense that in the case of partial subdivision networks we only subdivide some selected edges. Moreover, in this work we obtain the expressions for the group inverse, the effective resistances and the Kirchhoff Index associated with singular Shcrödinger operators on the partial subdivided network as a function of the corresponding parameters in the base network. Our approach consists in interpreting a network as an electric circuit, and hence each selected edge has got assigned a positive number that corresponds with the conductance of a wire connecting two nodes. In addition, we also consider a weight in the set of vertices, that reflects the relevance of each node. When we perform the subdivision operation we interpret that 
we introduce a rheostat in every subdivided edge, that is a device that may change the resistance without opening the circuit in which it is connected. Thus, we decompose each subdivided edge in two new edges taking into account electrical compatibility of the circuit, specifically, the series sum rule for resistances. As a consequence, we would get that after the subdivision process, the effective resistance between any pair of old vertices should remain unchanged except for a normalization factor.

The results obtained here include the ones obtained in [6], that correspond to the case of constant weight on the vertex set and when all the edges have been subdivided.

In the whole work, a network is the triplet $\Gamma=(V, E, c)$ where $(V, E)$ stands for a finite and connected graph, without loops nor multiple edges; and $c: V \times V \longrightarrow[0,+\infty)$ is a symmetric function called conductance satisfying $c(x, y)>0$ iff $x \sim y$ which means that $\{x, y\} \in E$. Let $n$ be the number of nodes and $m$ the number of edges.

On the other hand, $\mathcal{C}(V)$ is the set of real functions on $V$. For any vertex $x \in V, \varepsilon_{x} \in \mathcal{C}(V)$ is the Dirac function at $x$ and $k \in \mathcal{C}(V)$ defined as $k(x)=\sum_{y \in V} c(x, y)$, is the degree of $x$. The standard inner product in $\mathcal{C}(V)$ is denoted by $\langle\cdot, \cdot\rangle$; that is, if $u, v \in \mathcal{C}(V)$ then, $\langle u, v\rangle=\sum_{x \in V} u(x) v(x)$. A real-valued function $\omega \in \mathcal{C}(V)$ is called weight if $\omega(x)>0$ for any $x \in V$ and in addition $\|\omega\|=1$. The sets of weights on $V$ is denoted by $\Omega(V)$. Clearly the unique constant weight on $V$ assigns $\frac{1}{\sqrt{n}}$, to any vertex of $V$.

The combinatorial Laplacian or simply the Laplacian of the network $\Gamma$ is the endomorphism of $\mathcal{C}(V)$ that assigns to each $u \in \mathcal{C}(V)$ the function

$$
\mathcal{L}(u)(x)=\sum_{y \in V} c(x, y)(u(x)-u(y)), \quad x \in V .
$$

Given $q \in \mathcal{C}(V)$, the Schrödinger operator on $\Gamma$ with potential $q$ is the endomorphism of $\mathcal{C}(V)$ that assigns to each $u \in \mathcal{C}(V)$ the function $\mathcal{L}_{q}(u)=\mathcal{L}(u)+q u$, where $q u \in \mathcal{C}(V)$ is defined as $(q u)(x)=q(x) u(x)$; see for instance [1,3]. If $\omega$ is a weight, then the potential $q_{\omega}=-\omega \mathcal{L}^{-1}(\omega)$ is called potential determined by $\omega$. The Doob transform consists in the identity

$$
\mathcal{L}_{q_{\omega}}(u)(x)=\frac{1}{\omega(x)} \sum_{y \in V} c(x, y) \omega(x) \omega(y)\left(\frac{u(x)}{\omega(x)}-\frac{u(y)}{\omega(y)}\right), x \in V, u \in \mathcal{C}(V) .
$$

It is well-known that any Schrödinger operator is self-adjoint and moreover it is positive semidefinite iff there exist $\omega \in \Omega(V)$ and $\lambda \geq 0$ such that $q=q_{\omega}+\lambda$; see [1]. In addition, $\mathcal{L}_{q}$ is singular iff $\lambda=0$, in which case $\left\langle\mathcal{L}_{q_{\omega}}(v), v\right\rangle=0$ iff $v=a \omega, a \in \mathbb{R}$. In any case, $\lambda$ is the lowest eigenvalue of $\mathcal{L}_{q}$ and its associated eigenfunctions are multiple of $\omega$.

Throughout this work we will consider only positive semi-definite and singular Schrödinger operator, $\mathcal{L}_{q_{\omega}}$. Then, the operator that assigns to each function $f \in \mathcal{C}(V)$ the unique $u \in \mathcal{C}(V)$ such that $\mathcal{L}_{q_{\omega}}(u)=f-\langle\omega, f\rangle \omega$ and $\langle u, \omega\rangle=0$ is called Green's operator. The Green operator is denoted by $\mathscr{G}_{q_{\omega}}$, see [2]. Moreover, the function $G_{q_{\omega}}: V \times V \longrightarrow \mathbb{R}$, defined as $G_{q_{\omega}}(x, y)=\mathscr{G}_{q_{\omega}}\left(\varepsilon_{y}\right)(x)$, for any $x, y \in V$, is called Green's function. Observe that $\mathscr{G}_{q_{\omega}}(\omega)=0$, and moreover, $\mathscr{G}_{q_{\omega}}$ is self-adjoint as a consequence of the Fredholm Alternative and $\mathscr{G}_{q_{\omega}}$ is a symmetric function.

In [2], the authors introduced a generalization of the concept of effective resistance with respect 
to a weight $\omega \in \Omega(V)$. Specifically, from the functional on $\mathcal{C}(V)$ defined as

$$
\mathfrak{J}_{x, y}(u)=2\left[\frac{u(x)}{\omega(x)}-\frac{u(y)}{\omega(y)}\right]-\left\langle\mathcal{L}_{q_{\omega}}(u), u\right\rangle,
$$

we defined the generalization of the effective resistance.

Definition 1.1. Given $x, y \in V$, the effective resistance between $x$ and $y$ with respect to $\omega$, is the value

$$
R_{\omega}(x, y)=\max _{u \in \mathcal{C}(V)}\left\{\mathfrak{J}_{x, y}(u)\right\}
$$

When $\omega$ is constant we omit the subindex $\omega$. Therefore, $R$ is nothing else than a multiple of the standard effective resistance of the network.

The following result can be found in [2] and allows us to express the effective resistances in terms of the solution of a Poisson equation. In particular, these expressions will be useful to prove the main properties of the effective resistances.

Proposition 1.2. If $u \in \mathcal{C}(V)$ is a solution of the Poisson equation $\mathcal{L}_{q_{\omega}}(u)=\omega^{-1}\left(\varepsilon_{x}-\varepsilon_{y}\right)$, then

$$
R_{\omega}(x, y)=\left\langle\mathcal{L}_{q_{\omega}}(u), u\right\rangle=\frac{u(x)}{\omega(x)}-\frac{u(y)}{\omega(y)} .
$$

Therefore, $R_{\omega}$ is symmetric, non-negative and moreover $R_{\omega}(x, y)=0$ iff $x=y$. In addition,

$$
R_{\omega}(x, y)=\frac{G_{q_{\omega}}(x, x)}{\omega^{2}(x)}+\frac{G_{q_{\omega}}(y, y)}{\omega^{2}(y)}-\frac{2 G_{q_{\omega}}(x, y)}{\omega(x) \omega(y)} .
$$

Notice that, if we label the vertices of $\Gamma$, both the Schrödinger operator and the Green operator can be interpreted as singular matrices and hence, the Green kernel can be identified as the group inverse of the matrix associated with the Schrödinger operator.

The Kirchhoff Index of a network $\Gamma$ with respect to a weight is defined as

$$
\mathrm{k}_{\omega}(\Gamma)=\frac{1}{2} \sum_{x, y \in V} R_{\omega}(x, y) \omega^{2}(x) \omega^{2}(y)=\sum_{x \in V} G_{q_{\omega}}(x, x)
$$

and gives a measure of the global connectivity of the network. The Kirchhoff index is a descriptor of the structure of the network and exhibits many interesting interpretations, see [10, 14].

\section{The Poisson Problem on Partial Subdivision Networks}

Our objective in this section is to relate the solution of any Poisson problem on the partial subdivision network with the solution of an appropriate Poisson problem on the base network. In that way, we can obtain the solution of problems that have more variables in terms of problems of small size. In particular, we can obtain the group inverse of a big matrix in terms of the group inverse of a matrix with smaller size.

A partial subdivision network $\Gamma^{S}=\left(V^{S}, E^{S}, c^{S}\right)$ of a given network $\Gamma=(V, E, c)$, is obtained by inserting a new vertex in some edges of $\Gamma$, we denote the set of subdivided edges by $E^{\prime}$, so that 
each edge $\{x, y\} \in E^{\prime}$ is replaced by two new edges, say $\left\{x, v_{x y}\right\}$ and $\left\{y, v_{x y}\right\}$ where $v_{x y}$ is the new inserted vertex. We denote by $V^{\prime}$ the new vertex set assuming that, $v_{x y}=v_{y x}$. Thus, $V^{S}=V \cup V^{\prime}$, the order of the subdivision network is $n+\left|E^{\prime}\right|$, whereas the size is $m+\left|E^{\prime}\right|$. When, $E^{\prime}=E$, the partial subdivision network is nothing else but the so-called subdivision network; see [6]. Given $x \in V$ we denote by $S(x)$ the set of adjacent vertices to $x$ such that $\{x, y\} \in E^{\prime}$. Given $\omega: V \rightarrow \mathbb{R}^{+}$

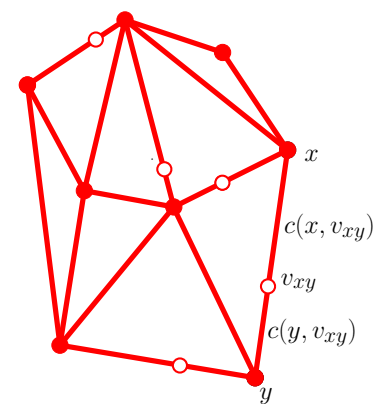

Figure 1: A partial subdivision network

a weight; that is, $\omega(x)>0$ and $\sum_{x \in V} \omega^{2}(x)=1$ in the base network, we now define an extension of this weight function, $\omega^{S}: V \cup V^{\prime} \rightarrow \mathbb{R}^{+}$in such a way that $\omega^{S}(x)=\alpha \omega(x)$ when $x \in V$ and $\omega^{S}\left(v_{x y}\right)=\alpha \omega\left(v_{x y}\right)$, where $\omega\left(v_{x y}\right)$ is absolutely arbitrary, except for positivity, for $v_{x y} \in V^{\prime}$ and

$$
\alpha^{2}=\frac{1}{1+\sum_{x \in V^{\prime}} \omega\left(v_{x y}\right)^{2}} .
$$

Moreover, according to the well-known rule that express the equivalent resistance of two resistors connected in series and the expression for the Schrödinger operator, we define the conductance function $c^{S}: V^{S} \times V^{S} \longrightarrow[0,+\infty)$ by choosing, for every edge in $E^{\prime},\{x, y\}$, non-null values $c^{S}\left(x, v_{x y}\right)$ and $c^{S}\left(y, v_{x y}\right)$ such that

$$
\frac{1}{\omega(x) \omega(y)} \frac{1}{c(x, y)}=\frac{1}{\omega(x) \omega\left(v_{x y}\right)} \frac{1}{c^{S}\left(x, v_{x y}\right)}+\frac{1}{\omega(y) \omega\left(v_{x y}\right)} \frac{1}{c^{S}\left(y, v_{x y}\right)},
$$

whereas for every edge in $E^{S} \backslash E^{\prime}$ we define $c^{S}(x, y)=c(x, y)$. The definition of $c^{S}$ cannot be misunderstood as all the edges in $E^{\prime}$ have both kind of vertices, one in $V$ and the other in $V^{\prime}$. Hence, by the sake of simplicity, it will be denoted as $c$. Moreover for each edge, there exist infinitely many different choices of conductances fulfilling (4), so that different choices will lead to different partial subdivision networks.

In the literature it has been studied the case of subdivision networks for the combinatorial Laplacian when $c(x, y)=c\left(x, v_{x y}\right)=c\left(y, v_{x y}\right)=1$, that not fulfills the electrical compatibility condition $(4)$, see $([7,9,13,15])$; and the case of arbitrary conductances when all the edges have been divided, see [6].

Observe that $\Gamma^{S}$ is also a connected, finite, with no loops, nor multiple edges network.

If $\mathcal{L}^{S}$ denotes the combinatorial Laplacian of $\Gamma^{S}$, then for any $u \in \mathcal{C}\left(V^{S}\right)$ we have that

$$
\begin{aligned}
\mathcal{L}^{S}(u)(x) & =\sum_{y \in V \backslash S(x)} c(x, y)(u(x)-u(y))+\sum_{y \in S(x)} c\left(x, v_{x y}\right)\left(u(x)-u\left(v_{x y}\right)\right), & & \text { for any } x \in V ; \\
\mathcal{L}^{S}(u)\left(v_{x y}\right) & =c\left(x, v_{x y}\right)\left(u\left(v_{x y}\right)-u(x)\right)+c\left(y, v_{x y}\right)\left(u\left(v_{x y}\right)-u(y)\right), & & \text { for any } v_{x y} \in V^{\prime} .
\end{aligned}
$$


On the other hand, we consider the potential determined by $\omega^{S}$,

$$
q^{\prime}=-\left(\omega^{S}\right)^{-1} \mathcal{L}^{S}\left(\omega^{S}\right)=-\omega^{-1} \mathcal{L}^{S}(\omega)
$$

and hence

$$
\begin{aligned}
\mathcal{L}_{q^{\prime}}^{S}(u)\left(v_{x y}\right) & =\frac{c\left(v_{x y}, x\right) \omega(x)+c\left(v_{x y}, y\right) \omega(y)}{\omega\left(v_{x y}\right)} u\left(v_{x y}\right)-c\left(v_{x y}, x\right) u(x)-c\left(v_{x y}, y\right) u(y), v_{x y} \in V^{\prime} \\
\mathcal{L}_{q^{\prime}}^{S}(u)(x) & =\frac{1}{\omega(x)} \sum_{y \in S(x)} c\left(x, v_{x y}\right) \omega(x) \omega\left(v_{x y}\right)\left[\frac{u(x)}{\omega(x)}-\frac{u\left(v_{x y}\right)}{\omega\left(v_{x y}\right)}\right] \\
& +\frac{1}{\omega(x)} \sum_{y \in V \backslash S(x)} c(x, y) \omega(x) \omega(y)\left[\frac{u(x)}{\omega(x)}-\frac{u(y)}{\omega(y)}\right], x \in V .
\end{aligned}
$$

Therefore, for any $v_{x y} \in V^{\prime}$ and $u \in \mathcal{C}\left(V^{S}\right)$ we have that

$$
\frac{u\left(v_{x y}\right)}{\omega\left(v_{x y}\right)}=\frac{\mathcal{L}_{q^{\prime}}^{S}(u)\left(v_{x y}\right)+c\left(x, v_{x y}\right) u(x)+c\left(y, v_{x y}\right) u(y)}{c\left(x, v_{x y}\right) \omega(x)+c\left(y, v_{x y}\right) \omega(y)} .
$$

Keeping in mind the compatibility equation (4) we can rewritte the expression for $\mathcal{L}_{q^{\prime}}^{S}(u)(x)$ as

$$
\mathcal{L}_{q^{\prime}}^{S}(u)(x)=\mathcal{L}_{q_{\omega}}(u)(x)-\sum_{y \in S(x)} \frac{c\left(x, v_{x y}\right) \omega\left(v_{x y}\right)}{c\left(x, v_{x y}\right) \omega(x)+c\left(y, v_{x y}\right) \omega(y)} \mathcal{L}_{q^{\prime}}^{S}(u)\left(v_{x y}\right) .
$$

This expression suggests to call contraction of $h \in \mathcal{C}\left(V^{S}\right)$ the function of $\mathcal{C}(V), \underline{h}$, defined as

$$
\underline{h}(x)=h(x)+\sum_{y \in S(x)} \alpha(x, y) h\left(v_{x y}\right),
$$

where

$$
\alpha(x, y)=\frac{c\left(x, v_{x y}\right) \omega\left(v_{x y}\right)}{c\left(x, v_{x y}\right) \omega(x)+c\left(y, v_{x y}\right) \omega(y)}
$$

Observe that,

$$
\alpha(x, y) \omega(x)+\alpha(y, x) \omega(y)=\omega\left(v_{x y}\right) .
$$

Moreover, we call extension of $u \in \mathcal{C}(V)$ with respect to $h \in \mathcal{C}\left(V^{S}\right)$, the function of $\mathcal{C}\left(V^{S}\right), u^{h}$, defined as

$$
\begin{aligned}
u^{h}\left(v_{x y}\right) & =\frac{h\left(v_{x y}\right) c(x, y)}{c\left(x, v_{x y}\right) c\left(y, v_{x y}\right)}+\alpha(x, y) u(x)+\alpha(y, x) u(y), v_{x y} \in V^{\prime} \\
u^{h}(x) & =u(x), x \in V
\end{aligned}
$$

Using these definitions we obtain from (5) that for any $u \in \mathcal{C}(V)$ and $x \in V$,

$$
\mathcal{L}_{q_{\omega}}(u)(x)=\underline{\mathcal{L}_{q^{\prime}}^{S}(u)}(x) .
$$

This relation allows us to obtain the following result.

Theorem 2.1. Given $h \in \mathcal{C}\left(V^{S}\right)$ such that $\left\langle h, \omega^{S}\right\rangle=0$, then $\langle\underline{h}, \omega\rangle=0$. Moreover, $\bar{u} \in \mathcal{C}\left(V^{S}\right)$ is a solution of the Poisson equation $\mathcal{L}_{q^{\prime}}^{S}(\bar{u})=h$ in $V^{S}$ iff $u=\bar{u}_{\mid V}$ is a solution of the Poisson equation $\mathcal{L}_{q_{\omega}}(u)=\underline{h}$ in $V$. In this case, the identity $\bar{u}=u^{h}$ holds. 
Proof. We only have to prove the first statement. For that we show that $\alpha\langle\underline{h}, \omega\rangle=\left\langle h, \omega^{S}\right\rangle$ as

$$
\begin{aligned}
\sum_{x \in V} \underline{h}(x) \omega(x) & =\sum_{x \in V} h(x) \omega(x)+\sum_{x \in V} \sum_{y \in S(x)} \alpha(x, y) h\left(v_{x y}\right) \omega(x) \\
& =\frac{1}{\alpha}\left(\sum_{x \in V} h(x) \omega^{S}(x)+\sum_{v_{x y} \in V^{\prime}} h\left(v_{x y}\right) \omega^{S}\left(v_{x y}\right)\right) .
\end{aligned}
$$

Next result shows how to obtain the unique solution of a Poisson problem on the partial subdivision network $\Gamma^{S}$ orthogonal to $\omega^{S}$.

Corollary 2.2. Given $h \in \mathcal{C}\left(V^{S}\right)$, such that $\left\langle h, \omega^{S}\right\rangle=0$, let $\underline{h} \in \mathcal{C}(V)$ be its contraction to $V$, $u \in \mathcal{C}(V)$ be the unique solution of $\mathcal{L}_{q_{\omega}}(u)=\underline{h}$ that satisfies $\langle u, \omega\rangle=0$ and the constant

$$
\lambda=-\sum_{\{x, y\} \in E^{\prime}} \frac{c(x, y) \omega^{S}\left(v_{x y}\right)}{c\left(x, v_{x y}\right) c\left(y, v_{x y}\right)}\left(h\left(v_{x y}\right)+c\left(x, v_{x y}\right) u(x)+c\left(y, v_{x y}\right) u(y)\right)
$$

Then, $u^{\perp}=u^{h}+\lambda \omega^{S}$ is the unique solution of $\mathcal{L}_{q^{\prime}}^{S}\left(u^{\perp}\right)=h$ that satisfies $\left\langle u^{\perp}, \omega^{S}\right\rangle=0$.

Proof. As two solutions differ on a constant, we have that $u^{\perp}=u^{h}+\gamma \omega^{S}, \gamma \in \mathbb{R}$. Then,

$$
\begin{aligned}
0 & =\left\langle u^{\perp}, \omega^{S}\right\rangle=\left\langle u^{h}, \omega^{S}\right\rangle+\gamma=\alpha \sum_{x \in V} u(x) \omega(x)+\sum_{v_{x y} \in V^{\prime}} u^{h}\left(v_{x y}\right) \omega^{S}\left(v_{x y}\right)+\gamma \\
& =\sum_{\{x, y\} \in E^{\prime}} \frac{h\left(v_{x y}\right) c(x, y)}{c\left(x, v_{x y}\right) c\left(y, v_{x y}\right)} \omega^{S}\left(v_{x y}\right)+\sum_{\{x, y\} \in E^{\prime}}(\alpha(x, y) u(x)+\alpha(y, x) u(y)) \omega^{S}\left(v_{x y}\right)+\gamma \\
& =\sum_{\{x, y\} \in E^{\prime}} \frac{h\left(v_{x y}\right) c(x, y)}{c\left(x, v_{x y}\right) c\left(y, v_{x y}\right)} \omega^{S}\left(v_{x y}\right)+\sum_{\{x, y\} \in E^{\prime}} c(x, y) \omega^{S}\left(v_{x y}\right)\left(\frac{u(x)}{c\left(y, v_{x y}\right)}+\frac{u(y)}{c\left(x, v_{x y}\right)}\right)+\gamma,
\end{aligned}
$$

because $\langle u, \omega\rangle=0$, and the result follows taking $\gamma=\lambda$.

The preceding results allows us to obtain the expression for the Green kernel of a partial subdivision network in terms of the Green kernel of the base network and some other parameters.

If we let

$$
\pi^{S}(x)=\sum_{y \sim S(x)} \frac{c(x, y) \omega^{S}\left(v_{x y}\right)}{c\left(y, v_{x y}\right)}=\sum_{y \sim S(x)} \alpha(x, y) \omega^{S}\left(v_{x y}\right)
$$

and

$$
\beta=\sum_{r, s \in V} G_{q_{\omega}}(s, r) \pi^{S}(r) \pi^{S}(s)+\sum_{\{r, s\} \in E^{\prime}} \frac{c(r, s) \omega^{S}\left(v_{r s}\right)^{2}}{c\left(r, v_{r s}\right) c\left(s, v_{r s}\right)},
$$

we get, in the next result, the desired expression.

Proposition 2.3. Let $\Gamma^{S}$ be the partial subdivision network of $\Gamma$, then for any $x, z \in V$ and 
$v_{x y}, v_{z t} \in V^{\prime}$, the Green kernel of $\Gamma^{S}$ is given by

$$
\begin{aligned}
G_{q^{\prime}}^{S}(x, z) & =G_{q_{\omega}}(x, z)-\sum_{\ell \in V}\left[\omega^{S}(z) G_{q_{\omega}}(x, \ell)+\omega^{S}(x) G_{q_{\omega}}(z, \ell)\right] \pi^{S}(\ell)+\beta \omega^{S}(x) \omega^{S}(z), \\
G_{q^{\prime}}^{S}\left(v_{x y}, z\right)= & \alpha(x, y) G_{q_{\omega}}(x, z)+\alpha(y, x) G_{q_{\omega}}(y, z) \\
& -\sum_{\ell \in V}\left(\omega^{S}(z) \alpha(x, y) G_{q_{\omega}}(x, \ell)+\omega^{S}(z) \alpha(y, x) G_{q_{\omega}}(y, \ell)+\omega^{S}\left(v_{x y}\right) G_{q_{\omega}}(z, \ell)\right) \pi^{S}(\ell) \\
& +\left(\beta-\frac{c(x, y)}{c\left(x, v_{x y}\right) c\left(y, v_{x y}\right)}\right) \omega^{S}\left(v_{x y}\right) \omega^{S}(z), \\
G_{q^{\prime}}^{S}\left(v_{x y}, v_{z t}\right)= & \frac{\varepsilon_{z t}\left(v_{x y}\right) c(x, y)}{c\left(x, v_{x y}\right) c\left(y, v_{x y}\right)}+\omega^{S}\left(v_{z t}\right) \omega^{S}\left(v_{x y}\right)\left(\beta-\frac{c(x, y)}{c\left(x, v_{x y}\right) c\left(y, v_{x y}\right)}-\frac{c(z, t)}{c\left(z, v_{z t}\right) c\left(t, v_{z t}\right)}\right) \\
& -\omega^{S}\left(v_{z t}\right) \sum_{\ell \in V}\left(\alpha(x, y) G_{q_{\omega}}(x, \ell)+\alpha(y, x) G_{q_{\omega}}(y, \ell)\right) \pi^{S}(\ell) \\
& -\omega^{S}\left(v_{x y}\right) \sum_{\ell \in V}\left(\alpha(z, t) G_{q_{\omega}}(z, \ell)+\alpha(t, z) G_{q_{\omega}}(t, \ell)\right) \pi^{S}(\ell) \\
& +\alpha(x, y)\left(\alpha(z, t) G_{q_{\omega}}(x, z)+\alpha(t, z) G_{q_{\omega}}(x, t)\right) \\
& +\alpha(y, x)\left(\alpha(z, t) G_{q_{\omega}}(y, z)+\alpha(t, z) G_{q_{\omega}}(y, t)\right) .
\end{aligned}
$$

Proof. Suppose $z \in V$, and let $h_{z}=\varepsilon_{z}-\omega^{S}(z) \omega^{S}$. Then, for every $x \in V$

$$
\begin{aligned}
\underline{h}_{z}(x) & =\varepsilon_{z}(x)-\omega^{S}(x) \omega^{S}(z)-\sum_{y \sim S(x)} \alpha(x, y) \omega^{S}\left(v_{x y}\right) \omega^{S}(z) \\
& =\varepsilon_{z}(x)-\omega^{S}(x) \omega^{S}(z)-\sum_{y \sim S(x)} \frac{c\left(x, v_{x y}\right) \omega^{S}\left(v_{x y}\right)}{c\left(x, v_{x y}\right) \omega(x)+c\left(y, v_{x y}\right) \omega(y)} \omega^{S}(z) \omega^{S}\left(v_{x y}\right) \\
& =\varepsilon_{z}(x)-\omega^{S}(x) \omega^{S}(z)-\omega^{S}(z) \sum_{y \sim S(x)} \frac{c(x, y) \omega^{S}\left(v_{x y}\right)}{c\left(y, v_{x y}\right)} \\
& =\varepsilon_{z}(x)-\left(\omega^{S}(x)+\pi^{S}(x)\right) \omega^{S}(z),
\end{aligned}
$$

where $\pi^{S}(x)=\sum_{y \sim S(x)} \frac{c(x, y) \omega^{S}\left(v_{x y}\right)}{c\left(y, v_{x y}\right)}$.

Hence, from Theorem 2.1, the Poisson problem to solve is $\mathcal{L}_{q_{\omega}}\left(u_{z}\right)=\underline{h}_{z}$, and, using the Green kernel for $\Gamma$, we obtain

$$
u_{z}(x)=G_{q_{\omega}}\left(\varepsilon_{z}\right)(x)-\sum_{\ell \in V} G_{q_{\omega}}(x, \ell) \pi^{S}(\ell) \omega^{S}(z)=G_{q_{\omega}}(x, z)-\sum_{\ell \in V} G_{q_{\omega}}(x, \ell) \pi^{S}(\ell) \omega^{S}(z) .
$$


Then, from Corollary 2.2

$$
\begin{aligned}
G_{q^{\prime}}^{S}\left(v_{x y}, z\right)= & \frac{h\left(v_{x y}\right) c(x, y)}{c\left(x, v_{x y}\right) c\left(y, v_{x y}\right)}+\alpha(x, y) u(x)+\alpha(y, x) u(y) \\
& -\sum_{\{r, s\} \in E^{\prime}} \frac{c(r, s) \omega^{S}\left(v_{r s}\right)}{c\left(r, v_{r s}\right) c\left(s, v_{r s}\right)}\left(h\left(v_{r s}\right)+c\left(r, v_{r s}\right) u(r)+c\left(s, v_{r s}\right) u(s)\right) \omega^{S}\left(v_{x y}\right) \\
= & -\frac{\omega^{S}\left(v_{x y}\right) \omega^{S}(z) c(x, y)}{c\left(x, v_{x y}\right) c\left(y, v_{x y}\right)}+\alpha(x, y) G_{q_{\omega}}(x, z)+\alpha(y, x) G_{q_{\omega}}(y, z) \\
& -\sum_{\ell \in V}\left(\omega^{S}(z) \alpha(x, y) G_{q_{\omega}}(x, \ell)+\omega^{S}(z) \alpha(y, x) G_{q_{\omega}}(y, \ell)+\omega^{S}\left(v_{x y}\right) G_{q_{\omega}}(z, \ell)\right) \pi^{S}(\ell) \\
& +\omega^{S}(z) \omega^{S}\left(v_{x y}\right) \sum_{\{r, s\} \in E^{\prime}} \frac{c(r, s) \omega^{S}\left(v_{r s}\right)}{c\left(r, v_{r s}\right) c\left(s, v_{r s}\right)} \omega^{S}\left(v_{r s}\right) \\
& +\omega^{S}(z) \omega^{S}\left(v_{x y}\right) \sum_{r, \ell \in V} G_{q_{\omega}}(r, \ell) \pi^{S}(\ell) \pi^{S}(r) .
\end{aligned}
$$

On the other hand,

$$
\begin{aligned}
G_{q^{\prime}}^{S}(x, z) & =u_{z}^{h_{z}}(x)-\sum_{\{r, s\} \in E^{\prime}} \frac{c(r, s) \omega^{S}\left(v_{r s}\right)}{c\left(r, v_{r s}\right) c\left(s, v_{r s}\right)}\left(h\left(v_{r s}\right)+c\left(r, v_{r s}\right) u(r)+c\left(s, v_{r s}\right) u(s)\right) \omega^{S}(x) \\
& =G_{q_{\omega}}(x, z)-\sum_{\ell \in V} G_{q_{\omega}}(x, \ell) \pi^{S}(\ell) \omega^{S}(z)+\omega^{S}(x) \omega^{S}(z) \sum_{\{r, s\} \in E^{\prime}} \frac{c(r, s) \omega^{S}\left(v_{r s}\right)^{2}}{c\left(r, v_{r s}\right) c\left(s, v_{r s}\right)} \\
& -\sum_{\{r, s\} \in E^{\prime}} \alpha(r, s)\left[G_{q_{\omega}}(r, z)-\sum_{\ell \in V} G_{q_{\omega}}(r, \ell) \pi^{S}(\ell) \omega^{S}(z)\right] \omega^{S}\left(v_{r s}\right) \omega^{S}(x) \\
& -\sum_{\{r, s\} \in E^{\prime}} \alpha(s, r)\left[G_{q_{\omega}}(s, z)-\sum_{\ell \in V} G_{q_{\omega}}(s, \ell) \pi^{S}(\ell) \omega^{S}(z)\right] \omega^{S}\left(v_{r s}\right) \omega^{S}(x) \\
& =G_{q_{\omega}}(x, z)-\omega^{S}(x) \omega^{S}(z) \sum_{\ell \in V}\left[\frac{G_{q_{\omega}}(x, \ell)}{\omega^{S}(x)}+\frac{G_{q_{\omega}}(z, \ell)}{\left.\omega^{S}(z)\right] \pi^{S}(\ell)}\right. \\
& +\omega^{S}(x) \omega^{S}(z) \sum_{r, s \in V} G_{q_{\omega}}(s, r) \pi^{S}(r) \pi^{S}(s) \\
& +\omega^{S}(x) \omega^{S}(z) \sum_{\{r, s\} \in E^{\prime}} \frac{c(r, s) \omega^{S}\left(v_{r s}\right)^{2}}{c\left(r, v_{r s}\right) c\left(s, v_{r s}\right)} .
\end{aligned}
$$


Suppose now $v_{z t} \in V^{\prime}$, and let $h_{v_{z t}}=\varepsilon_{v_{z t}}-\omega^{S}\left(v_{z t}\right) \omega^{S}$. Then, for every $x \in V$

$$
\begin{aligned}
\underline{h}_{v_{z t}}(x) & =\varepsilon_{v_{z t}}(x)-\omega^{S}\left(v_{z t}\right) \omega^{S}(x)+\sum_{y \in S(x)} \alpha(x, y)\left(\varepsilon_{v_{z t}}\left(v_{x y}\right)-\omega^{S}\left(v_{z t}\right) \omega^{S}\left(v_{x y}\right)\right) \\
& =-\omega^{S}\left(v_{z t}\right) \omega^{S}(x)+\sum_{y \in S(x)} \alpha(x, y)\left(\varepsilon_{v_{z t}}\left(v_{x y}\right)-\omega^{S}\left(v_{z t}\right) \omega^{S}\left(v_{x y}\right)\right) \\
& =-\omega^{S}\left(v_{z t}\right) \omega^{S}(x)+\sum_{y \in S(x)} \alpha(x, y) \varepsilon_{v_{z t}}\left(v_{x y}\right)-\omega^{S}\left(v_{z t}\right) \sum_{y \in S(x)} \alpha(x, y) \omega^{S}\left(v_{x y}\right) \\
& =-\omega^{S}\left(v_{z t}\right) \omega^{S}(x)+\alpha(z, t) \varepsilon_{z}(x)+\alpha(t, z) \varepsilon_{t}(x)-\omega\left(v_{z t}\right) \pi^{S}(x) \\
& =-\omega^{S}\left(v_{z t}\right)\left(\omega^{S}(x)+\pi^{S}(x)\right)+\alpha(z, t) \varepsilon_{z}(x)+\alpha(t, z) \varepsilon_{t}(x) .
\end{aligned}
$$

Hence, the Poisson problem to solve is $\mathcal{L}_{q_{\omega}}\left(u_{v_{z t}}\right)=\underline{h}_{v_{z t}}$, and, using Green's kernel for $\Gamma$, we obtain

$$
\begin{aligned}
u_{v_{z t}}(x) & =-\omega^{S}\left(v_{z t}\right) \sum_{\ell \in V} G_{q_{\omega}}(x, \ell)\left(\omega(\ell)+\pi^{S}(\ell)\right)+\alpha(z, t) G_{q_{\omega}}(x, z)+\alpha(t, z) G_{q_{\omega}}(x, t) \\
& =-\omega^{S}\left(v_{z t}\right) \sum_{\ell \in V} G_{q_{\omega}}(x, \ell) \pi^{S}(\ell)+\alpha(z, t) G_{q_{\omega}}(x, z)+\alpha(t, z) G_{q_{\omega}}(x, t) .
\end{aligned}
$$

Then, the result follows by applying again Corollary 2.2 .

If we consider $E^{\prime}=E$; that is, the case of subdivision networks, the above result coincides except for a constant with [6, Proposition 3.1]. The scalar is due to the fact that in the mentioned work, we were considering no weights in the vertex set; i.e., $\omega(x)=1$ for any $x \in V$ and hence the normalization factor appears.

\section{Resistance distances}

In this section we aim at obtaining the expression for the effective resistances on a partial subdivision network of a given network $\Gamma$. The expression will follow by taking into account the expression for the effective resistances in terms of Green's function as stated in Proposition 1.2. Again, the results coincide except for a constant with [6, Proposition 4.1].

Proposition 3.1. Let $\Gamma^{S}$ be a partial subdivision network of $\Gamma$, then for any $x, z \in V$ and $v_{x y}, v_{z t} \in$ 
$V^{\prime}$, the Effective resistances of $\Gamma^{S}$ are given by

$$
\begin{aligned}
R_{\omega S}^{S}(x, z) & =\frac{1}{\alpha^{2}} R_{\omega}(x, y) \\
R_{\omega^{S}}^{S}\left(v_{z t}, x\right) & =\frac{c(z, t)}{\alpha^{2} c\left(z, v_{z t}\right) c\left(t, v_{z t}\right) \omega\left(v_{z t}\right)^{2}} \\
& +\frac{\omega(z) \omega(t)}{\alpha^{2} \omega\left(v_{z t}\right)}\left(\frac{\alpha(z, t) R_{\omega}(x, z)}{\omega(t)}+\frac{\alpha(t, z) R_{\omega}(x, t)}{\omega(z)}-\frac{\alpha(t, z) \alpha(z, t) R_{\omega}(z, t)}{\omega\left(v_{z t}\right)}\right) \\
R_{\omega^{S}}^{S}\left(v_{x y}, v_{z t}\right) & =\frac{c(x, y)}{\alpha^{2} c\left(x, v_{x y}\right) c\left(y, v_{x y}\right) \omega\left(v_{x y}\right)^{2}}+\frac{c(z, t)}{\alpha^{2} c\left(z, v_{z t}\right) c\left(t, v_{z t}\right) \omega\left(v_{z t}\right)^{2}} \\
& +\frac{1}{\alpha^{2} \omega\left(v_{x y}\right) \omega\left(v_{z t}\right)}\left[\alpha(x, y) \alpha(z, t) \omega(x) \omega(z) R_{\omega}(x, z)+\alpha(x, y) \alpha(t, z) \omega(x) \omega(t) R_{\omega}(x, t)\right. \\
& \left.+\alpha(y, x) \alpha(z, t) \omega(y) \omega(z) R_{\omega}(y, z)+\alpha(y, x) \alpha(t, z) \omega(y) \omega(t) R_{\omega}(y, t)\right] \\
& -\frac{\alpha(x, y) \alpha(y, x) \omega(x) \omega(y)}{\alpha^{2} \omega\left(v_{x y}\right)^{2}} R_{\omega}(x, y)-\frac{\alpha(z, t) \alpha(t, z) \omega(z) \omega(t)}{\alpha^{2} \omega\left(v_{z t}\right)^{2}} R_{\omega}(z, t), \text { for any } v_{x y} \neq v_{z t} .
\end{aligned}
$$

Proof. Suppose that $x, z \in V$, then

$$
\begin{aligned}
R_{\omega^{S}}^{S}(x, z) & =\frac{G_{q^{\prime}}^{S}(x, x)}{\left[\omega^{S}(x)\right]^{2}}+\frac{G_{q^{\prime}}^{S}(z, z)}{\left[\omega^{S}(z)\right]^{2}}-2 \frac{G_{q^{\prime}}^{S}(x, z)}{\omega^{S}(x) \omega^{S}(z)} \\
& =\frac{1}{\left[\omega^{S}(x)\right]^{2}}\left(G_{q_{\omega}}(x, x)-2 \omega^{S}(x) \sum_{\ell \in V} G_{q_{\omega}}(x, \ell) \pi^{S}(\ell)\right) \\
& +\frac{1}{\left[\omega^{S}(y)\right]^{2}}\left(G_{q_{\omega}}(y, y)-2 \omega^{S}(y) \sum_{\ell \in V} G_{q_{\omega}}(y, \ell) \pi^{S}(\ell)\right) \\
& -2 \frac{1}{\omega^{S}(x) \omega^{S}(y)}\left(G_{q_{\omega}}(x, y)-\sum_{\ell \in V}\left(\omega^{S}(x) G_{q_{\omega}}(y, \ell)+\omega^{S}(y) G_{q_{\omega}}(x, \ell)\right) \pi^{S}(\ell)\right) \\
& =\frac{G_{q_{\omega}}(x, x)}{\left[\omega^{S}(x)\right]^{2}}+\frac{G_{q_{\omega}}(y, y)}{\left[\omega^{S}(y)\right]^{2}}-2 \frac{G_{q_{\omega}}(x, y)}{\omega^{S}(x) \omega^{S}(y)}=\frac{1}{\alpha^{2}} R_{\omega}(x, y) .
\end{aligned}
$$

Moreover, if $x \in V$ and $v_{z t} \in V^{\prime}$, then

$$
R_{q^{\prime}}^{S}\left(x, v_{z t}\right)=\frac{G_{q^{\prime}}^{S}(x, x)}{\left[\omega^{S}(x)\right]^{2}}+\frac{G_{q^{\prime}}^{S}\left(v_{z t}, v_{z t}\right)}{\left[\omega^{S}\left(v_{z t}\right)\right]^{2}}-2 \frac{G_{q^{\prime}}^{S}\left(x, v_{z t}\right)}{\omega^{S}(x) \omega^{S}\left(v_{z t}\right)}
$$


where from Proposition 2.3

$$
\begin{aligned}
\frac{G_{q_{\omega}}^{S}(x, x)}{\omega^{S}(x)^{2}} & =\frac{G_{q_{\omega}}(x, x)}{\omega^{S}(x)^{2}}-\frac{2}{\omega^{S}(x)} \sum_{\ell \in V} G_{q_{\omega}}(x, \ell) \pi^{S}(\ell)+\beta \\
\frac{G_{q^{\prime}}^{S}\left(v_{z t}, v_{z t}\right)}{\omega^{S}\left(v_{z t}\right)^{2}} & =\frac{c(z, t)}{c\left(z, v_{z t}\right) c\left(t, v_{z t}\right) \omega^{S}\left(v_{z t}\right)^{2}}-2 \frac{c(z, t)}{c\left(z, v_{z t}\right) c\left(t, v_{z t}\right)} \\
& -\frac{2}{\omega^{S}\left(v_{z t}\right)} \sum_{\ell \in V}\left(\alpha(z, t) G_{q_{\omega}}(z, \ell)+\alpha(t, z) G_{q_{\omega}}(t, \ell)\right) \pi^{S}(\ell) \\
& +\frac{\alpha(z, t)^{2} G_{q_{\omega}}(z, z)+2 \alpha(z, t) \alpha(t, z) G_{q_{\omega}}(z, t)+\alpha(t, z)^{2} G_{q_{\omega}}(t, t)}{\omega^{S}\left(v_{z t}\right)^{2}}+\beta \\
\frac{G_{q^{\prime}}^{S}\left(v_{z t}, x\right)}{\omega^{S}\left(v_{z t}\right) \omega^{S}(x)} & =\frac{\alpha(z, t) G_{q_{\omega}}(z, x)+\alpha(t, z) G_{q_{\omega}}(t, x)}{\omega^{S}\left(v_{z t}\right) \omega^{S}(x)}-\frac{c(z, t)}{c\left(z, v_{z t}\right) c\left(t, v_{z t}\right)}+\beta \\
& -\sum_{\ell \in V}\left(\frac{\alpha(z, t) G_{q_{\omega}}(z, \ell)}{\omega^{S}\left(v_{z t}\right)}+\frac{\alpha(t, z) G_{q_{\omega}}(t, \ell)}{\omega^{S}\left(v_{z t}\right)}+\frac{G_{q_{\omega}}(x, \ell)}{\omega^{S}(x)}\right) \pi^{S}(\ell) .
\end{aligned}
$$

Summing up

$$
\begin{aligned}
R_{\omega^{s}}^{S}\left(x, v_{z t}\right) & =\frac{c(z, t)}{c\left(z, v_{z t}\right) c\left(t, v_{z t}\right) \omega^{S}\left(v_{z t}\right)^{2}}+\frac{G_{q_{\omega}}(x, x)}{\omega^{S}(x)^{2}}+\frac{\alpha(z, t)^{2} G_{q_{\omega}}(z, z)}{\omega^{S}\left(v_{z t}\right)^{2}}+\frac{\alpha(t, z)^{2} G_{q_{\omega}}(t, t)}{\omega^{S}\left(v_{z t}\right)^{2}} \\
& +\frac{2 \alpha(z, t) \alpha(t, z) G_{q_{\omega}}(z, t)}{\omega^{S}\left(v_{z t}\right)^{2}}-2 \frac{\alpha(z, t) G_{q_{\omega}}(z, x)+\alpha(t, z) G_{q_{\omega}}(t, x)}{\omega^{S}\left(v_{z t}\right) \omega^{S}(x)} \\
& =\frac{c(z, t)}{c\left(z, v_{z t}\right) c\left(t, v_{z t}\right) \omega^{S}\left(v_{z t}\right)^{2}} \\
& +\frac{G_{q_{\omega}}(x, x)}{\omega^{S}(x)^{2}}\left(\frac{\alpha(z, t) \omega(z)}{\omega\left(v_{z t}\right)}+\frac{\alpha(t, z) \omega(t)}{\omega\left(v_{z t}\right)}\right) \\
& +\frac{G_{q_{\omega}}(z, z)}{\omega^{S}(z)^{2}}\left(\frac{\alpha(z, t) \omega(z)}{\omega\left(v_{z t}\right)}-\frac{\alpha(t, z) \alpha(z, t) \omega(z) \omega(t)}{\omega\left(v_{z t}\right)^{2}}\right) \\
& +\frac{G_{q_{\omega}}(t, t)}{\omega^{S}(t)^{2}}\left(\frac{\alpha(t, z) \omega(t)}{\omega\left(v_{z t}\right)}-\frac{\alpha(t, z) \alpha(z, t) \omega(z) \omega(t)}{\omega\left(v_{z t}\right)^{2}}\right) \\
& +\frac{2 \alpha(z, t) \alpha(t, z) \omega(z) \omega(t)}{\omega^{S}\left(v_{z t}\right)^{2}} \frac{G_{q_{\omega}}(z, t)}{\omega(z) \omega(t)}-2 \frac{\alpha(z, t) \omega(z)}{\omega^{S}\left(v_{z t}\right)} \frac{G_{q_{\omega}}(z, x)}{\omega^{S}(x) \omega(z)}-2 \frac{\alpha(t, z) \omega(t)}{\omega^{S}\left(v_{z t}\right)} \frac{G_{q_{\omega}}(t, x)}{\omega^{S}(x) \omega(t)} \\
& =\frac{c(z, t)}{c\left(z, v_{z t}\right) c\left(t, v_{z t}\right) \omega^{S}\left(v_{z t}\right)^{2}} \\
& +\frac{\alpha(z, t) \omega(z)}{\alpha^{2} \omega\left(v_{z t}\right)} R_{\omega}(x, z)+\frac{\alpha(t, z) \omega(t)}{\alpha^{2} \omega\left(v_{z t}\right)} R_{\omega}(x, t)-\frac{\alpha(t, z) \alpha(z, t) \omega(z) \omega(t)}{\alpha^{2} \omega\left(v_{z t}\right)^{2}} R_{\omega}(z, t) .
\end{aligned}
$$

The last case, follows by performing similar calculations. 
Proposition 3.2. Let $\Gamma^{S}$ be the partial subdivision network of $\Gamma$, then the Kirchhoff index of $\Gamma^{S}$ is given by

$$
\begin{aligned}
\mathrm{k}_{\omega S}^{S}\left(\Gamma^{S}\right) & =\mathrm{k}_{\omega}(\Gamma)+\sum_{x \in V} \frac{\pi^{S}(x)}{\omega^{S}(x)} G_{q_{\omega}}(x, x)-\sum_{\{x, y\} \in E^{\prime}} \alpha(x, y) \alpha(y, x) \omega(x) \omega(y) R_{\omega}(x, y) \\
& +\sum_{\{x, y\} \in E^{\prime}} \frac{c(x, y)}{c\left(x, v_{x y}\right) c\left(y, v_{x y}\right)}-\beta .
\end{aligned}
$$

Proof. It is enough to compute the trace of Green's function.

$$
\begin{aligned}
& \mathrm{k}_{\omega^{S}}^{S}\left(\Gamma^{S}\right)=\sum_{x \in V}\left[G_{q_{\omega}}(x, x)-2 \omega^{S}(x) \sum_{\ell \in V} G_{q_{\omega}}(x, \ell) \pi^{S}(\ell)+\beta \omega^{S}(x)^{2}\right] \\
&+\sum_{\{x, y\} \in E^{\prime}}\left[\frac{c(x, y)}{c\left(x, v_{x y}\right) c\left(y, v_{x y}\right)}-\omega^{S}\left(v_{x y}\right)^{2}\left(2 \frac{c(x, y)}{c\left(x, v_{x y}\right) c\left(y, v_{x y}\right)}-\beta\right)\right. \\
&-2 \omega\left(v_{x y}\right) \sum_{\ell \in V}\left(\alpha(x, y) G_{q_{\omega}}(x, \ell)+\alpha(y, x) G_{q_{\omega}}(y, \ell)\right) \pi^{S}(\ell) \\
&\left.+\alpha(x, y)^{2} G_{q_{\omega}}(x, x)+2 \alpha(x, y) \alpha(y, x) G_{q_{\omega}}(x, y)+\alpha(y, x)^{2} G_{q_{\omega}}(y, y)\right] \\
&=\mathrm{k}_{\omega}(\Gamma)+\beta+\sum_{\{x, y\} \in E^{\prime}}\left[\frac{c(x, y)}{c\left(x, v_{x y}\right) c\left(y, v_{x y}\right)}-2 \frac{c(x, y) \omega^{S}\left(v_{x y}\right)^{2}}{c\left(x, v_{x y}\right) c\left(y, v_{x y}\right)}\right] \\
&-2 \sum_{x, \ell \in V} G_{q_{\omega}}(x, \ell) \pi^{S}(x) \pi^{S}(\ell) \\
&+\sum_{\{x, y\} \in E^{\prime}}\left[\alpha(x, y)^{2} G_{q_{\omega}}(x, x)+2 \alpha(x, y) \alpha(y, x) G_{q_{\omega}}(x, y)+\alpha(y, x)^{2} G_{q_{\omega}}(y, y)\right] \\
&=\mathrm{k}_{\omega}(\Gamma)+\sum_{x \in V} G_{q_{\omega}}(x, x) \frac{\pi^{S}(x)}{\omega^{S}(x)}-\sum_{\{x, y\} \in E^{\prime}} \alpha(x, y) \alpha(y, x) \omega(x) \omega(y) R_{\omega}(x, y) \\
&+\sum_{\{x, y\} \in E^{\prime}} \frac{c(x, y)}{c\left(x, v_{x y}\right) c\left(y, v_{x y}\right)}-\beta . \quad \square \\
& \square
\end{aligned}
$$

\section{Example: Partial subdivision of a Star}

Let us consider $S_{2 n}=(V, E, c)$, the Star network with vertex set $V=\left\{x_{0}, x_{1}, \ldots, x_{2 n}\right\}$ and positive conductances

$$
c\left(x_{0}, x_{2 j}\right)=a_{j}, \quad c\left(x_{0}, x_{2 j-1}\right)=a_{j}^{\prime}
$$

for $j=1, \ldots n$ and $c\left(x_{i}, x_{j}\right)=0$ otherwise. We define $S_{2 n}^{S}=\left(V \cup V^{\prime}, c^{S}\right)$, the partial subdivision network of $S_{2 n}$ where $V^{\prime}=\left\{x_{02 i}, \mid i=1, \ldots, n\right\}$ and

$$
c^{S}\left(x_{02 i}, x_{0}\right)=c_{0 i}>0, \quad c^{S}\left(x_{02 i}, x_{2 i}\right)=c_{i 0}>0
$$


for $i=1, \ldots, n$ and $c^{S}\left(x_{i}, x_{j}\right)=c\left(x_{i}, x_{j}\right)$ otherwise. See, Fig. 2 .
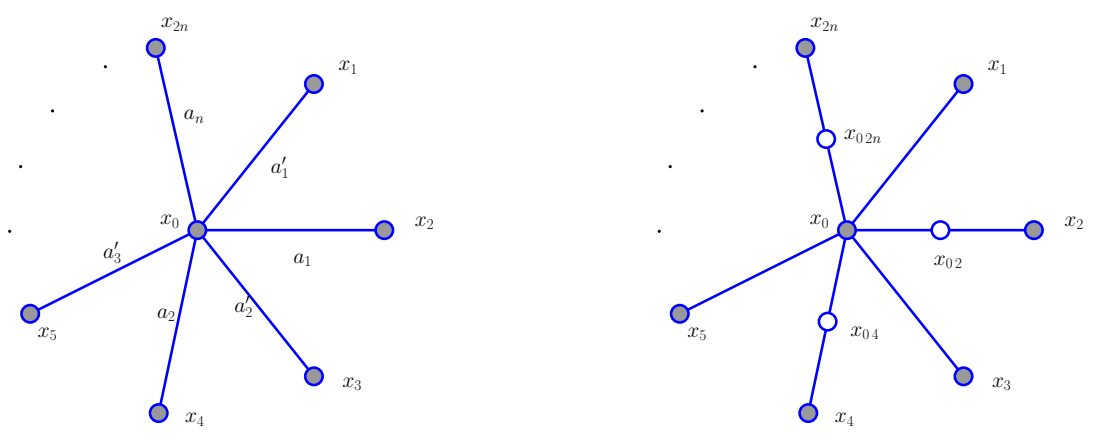

Figure 2: The Star network (left) and a partial subdivision (right)

Let $\omega: V \rightarrow \mathbb{R}^{+}$be a weight on $S_{2 n}$ and we define an extension of this weight function $\omega^{S}: V \cup V^{\prime} \rightarrow \mathbb{R}^{+}$as mentioned in the introduction. In order to simplify the notation, the weight function $S_{2 n}^{S}$ will be denoted

$$
\omega^{S}\left(x_{j}\right)=\omega_{j}, \quad \omega^{S}\left(x_{02 i}\right)=\omega_{02 i}
$$

for any $j=0, \ldots, 2 n, i=1, \ldots, n$. The compatibility condition (4) reads

$$
\frac{\omega_{02 i}}{a_{i}}=\frac{\omega_{2 i}}{c_{0 i}}+\frac{\omega_{0}}{c_{i 0}} \text {. }
$$

Let us assume constant weight for the Star network $\omega\left(x_{i}\right)=\frac{1}{\sqrt{2 n+1}}$, for any $i=0, \ldots, 2 n$, and we assign a positive constant $\gamma$ to the weight of the new vertices. Thus,

$$
\omega^{S}\left(x_{i}\right)=\omega_{i}=\frac{\alpha}{\sqrt{2 n+1}}, \quad \omega^{S}\left(x_{02 i}\right)=\omega_{02 i}=\alpha \gamma
$$

In this case, $\alpha^{2}=\frac{1}{1+n \gamma^{2}}$. Moreover, we assume equal conductance for each pair of new edges, this is, $c_{i 0}=c_{0} i$ for any $i=1, \ldots, n$. Then, according to expression (4), the following equality holds

$$
\frac{a_{i}}{c_{0 i}}=\frac{a_{i}}{c_{i 0}}=\frac{1}{2} \gamma \sqrt{2 n+1}
$$

Moreover, the parameters are

$$
\begin{aligned}
\alpha\left(x_{0}, x_{2 i}\right) & =\frac{c_{0 i} \omega_{02 i}}{c_{0 i} \omega_{0}+c_{i 0} \omega_{2 i}}=\frac{a_{i}}{c_{0 i}}=\frac{1}{2} \gamma \sqrt{2 n+1} \\
\pi^{S}\left(x_{0}\right) & =\sum_{i=1}^{n} \frac{c_{0 i} \omega_{02 i}^{2}}{c_{0 i} \omega_{0}+c_{i 0} \omega_{2 i}}=\sum_{i=1}^{n} \frac{a_{i} \omega_{02 i}}{c_{i 0}}=\frac{n}{2} \alpha \gamma^{2} \sqrt{2 n+1} \\
\pi^{S}\left(x_{2 i}\right) & =\frac{a_{i} \omega_{02 i}}{c_{0 i}}=\frac{1}{2} \alpha \gamma^{2} \sqrt{2 n+1} .
\end{aligned}
$$

If we denote

$$
Q=\frac{1}{2 n+1} \sum_{j=1}^{n}\left(\frac{1}{a_{j}}+\frac{1}{a_{j}^{\prime}}\right)
$$


the expression for the Green function and the effective resistance of the Star network is, see [5]

$$
\begin{array}{llrl}
G\left(x_{0}, x_{0}\right) & =\frac{1}{2 n+1} Q, & G\left(x_{\ell}, x_{k}\right) & =\frac{1}{2 n+1}\left(Q-\frac{1}{c\left(x_{0}, x_{\ell}\right)}-\frac{1}{c\left(x_{0}, x_{k}\right)}\right) \\
G\left(x_{0}, x_{k}\right) & =\frac{1}{2 n+1}\left(Q-\frac{1}{c\left(x_{0}, x_{k}\right)}\right), & G\left(x_{k}, x_{k}\right) & =\frac{1}{2 n+1}\left(Q-\frac{2}{c\left(x_{0}, x_{k}\right)}\right)+\frac{1}{c\left(x_{0}, x_{k}\right)},
\end{array}
$$

and

$$
R\left(x_{0}, x_{k}\right)=\frac{2 n+1}{c\left(x_{0}, x_{k}\right)}, \quad R\left(x_{\ell}, x_{k}\right)=(2 n+1)\left(\frac{1}{c\left(x_{0}, x_{\ell}\right)}+\frac{1}{c\left(x_{0}, x_{k}\right)}\right),
$$

where $\ell \neq k$ and $l, k=1, \ldots, 2 n$.

Moreover, under the previous assumptions, the expression for the Kirchhoff index is

$$
\mathrm{k}\left(S_{2 n}\right)=2 n Q .
$$

In order to obtain the Green function for the partial subdivision network of the Star, we first compute $\beta$, that in this case is

$$
\begin{aligned}
\beta & =G\left(x_{0}, x_{0}\right) \pi\left(x_{0}\right)^{2}+2 \sum_{i=1}^{n} G\left(x_{0}, x_{2 i}\right) \pi\left(x_{0}\right) \pi\left(x_{2 i}\right) \\
& +\sum_{i, j=1}^{n} G\left(x_{2 i}, x_{2 j}\right) \pi\left(x_{2 i}\right) \pi\left(x_{2 j}\right)+\sum_{i=1}^{n} \frac{a_{i} \omega_{02 i}^{2}}{c(0,2 i) c(2 i, 0)} \\
& =\frac{1}{4} n^{2} \alpha^{2} \gamma^{4} Q+\frac{2}{4} n^{2} \alpha^{2} \gamma^{4} Q-\frac{2 n}{4} \alpha^{2} \gamma^{4} \sum_{i=1}^{n} \frac{1}{a_{i}}+\frac{2}{4} \alpha^{2} \gamma^{4} \sum_{i<j, i=1}^{n}\left[Q-\frac{1}{a_{i}}-\frac{1}{a_{j}}\right] \\
& +\frac{1}{4} \alpha^{2} \gamma^{4} \sum_{i=1}^{n}\left[Q-\frac{2}{a_{i}}\right]+\frac{2 n+1}{4} \alpha^{2} \gamma^{2} \sum_{i=1}^{n} \frac{1}{a_{i}}+\sum_{i=1}^{n} \frac{a_{i} \omega_{02 i}^{2}}{c(0,2 i) c(2 i, 0)} \\
& =n^{2} \alpha^{2} \gamma^{4} Q+\frac{\alpha^{2} \gamma^{4}}{2} \sum_{i=1}^{n} \frac{1}{a_{i}} .
\end{aligned}
$$

Proposition 4.1. The Green function for the partial subdivision network of the Star network $S_{2 n}$ 
has the following expression according to the different vertices involved

$$
\begin{aligned}
G_{\omega^{S}}^{S}\left(x_{0}, x_{0}\right) & =\frac{\alpha^{4}}{2 n+1} Q+\alpha^{2} \gamma^{2} \mathrm{f}_{\mathrm{ev}} \sum_{i=1}^{n} \frac{1}{a_{i}}, \\
G_{\omega^{S}}^{S}\left(x_{0}, x_{2 i}\right) & =G_{\omega^{S}}^{S}\left(x_{0}, x_{0}\right)-\mathrm{f}_{\mathrm{ev}} \frac{1}{a_{i}}, \quad G_{\omega^{S}}^{S}\left(x_{0}, x_{2 i-1}\right)=G_{\omega^{S}}^{S}\left(x_{0}, x_{0}\right)-\mathrm{f}_{\mathrm{odd}} \frac{1}{a_{i}^{\prime}}, \\
G_{\omega^{S}}^{S}\left(x_{2 i}, x_{2 j}\right) & =G_{\omega^{S}}^{S}\left(x_{0}, x_{0}\right)+\varepsilon_{x_{2 i}}\left(x_{2 j}\right) \frac{1}{a_{i}}-\mathrm{f}_{\mathrm{ev}}\left(\frac{1}{a_{i}}+\frac{1}{a_{j}}\right) \\
G_{\omega^{S}}^{S}\left(x_{2 i}, x_{2 j-1}\right) & =G_{\omega^{S}}^{S}\left(x_{0}, x_{0}\right)-\mathrm{f}_{\mathrm{ev}} \frac{1}{a_{i}}-\mathrm{f}_{\mathrm{odd}} \frac{1}{a_{j}^{\prime}}, \\
G_{\omega^{S}}^{S}\left(x_{2 i-1}, x_{2 j-1}\right) & =G_{\omega^{S}}^{S}\left(x_{0}, x_{0}\right)+\varepsilon_{x_{2 i}}\left(x_{2 j}\right) \frac{1}{a_{i}^{\prime}}-\mathrm{f}_{\mathrm{odd}}\left(\frac{1}{a_{i}^{\prime}}+\frac{1}{a_{j}^{\prime}}\right), \\
G_{\omega^{S}}^{S}\left(x_{0}, x_{02 i}\right) & =\gamma \sqrt{2 n+1}\left(G_{\omega^{S}}^{S}\left(x_{0}, x_{0}\right)-\mathrm{f}_{\mathrm{sub}} \frac{1}{a_{i}}\right), \\
G_{\omega^{S}}^{S}\left(x_{2 i-1}, x_{02 j}\right) & =\gamma \sqrt{2 n+1}\left(G_{\omega^{S}}^{S}\left(x_{0}, x_{0}\right)-\mathrm{f}_{\mathrm{odd}} \frac{1}{a_{i}^{\prime}}-\mathrm{f}_{\mathrm{sub}} \frac{1}{a_{j}}\right), \\
G_{\omega^{S}}^{S}\left(x_{2 i}, x_{02 j}\right) & =\gamma \sqrt{2 n+1}\left(G_{\omega^{S}}^{S}\left(x_{0}, x_{0}\right)+\frac{1}{2} \varepsilon_{x_{2 i}}\left(x_{2 j}\right) \frac{1}{a_{j}}-\mathrm{f}_{\mathrm{ev}} \frac{1}{a_{i}}-\mathrm{f}_{\mathrm{sub}} \frac{1}{a_{j}}\right) \\
G_{\omega^{S}}^{S}\left(x_{02 i}, x_{02 j}\right) & =\gamma^{2}(2 n+1)\left(G_{\omega^{S}}^{S}\left(x_{0}, x_{0}\right)+\frac{1}{2} \varepsilon_{x_{2 i}}\left(x_{2 j}\right) \frac{1}{a_{j}}-\mathrm{f}_{\mathrm{sub}}\left(\frac{1}{a_{i}}+\frac{1}{a_{j}}\right)\right)
\end{aligned}
$$

where

$$
\mathrm{f}_{\mathrm{odd}}=\frac{\alpha^{2}}{2 n+1}, \quad \mathrm{f}_{\mathrm{ev}}=\frac{2+\alpha^{2} \gamma^{2}}{2(2 n+1)} \quad \text { and } \quad \mathrm{f}_{\mathrm{sub}}=\frac{1+(n+1) \alpha^{2} \gamma^{2}}{2(2 n+1)}
$$

Proof. We compute only some cases by using the results given in Proposition 2.3.

$$
\begin{aligned}
G_{\omega^{S}}^{S}\left(x_{0}, x_{0}\right) & =G\left(x_{0}, x_{0}\right)-2 \sum_{\ell \in V} \omega_{0} G\left(x_{0}, x_{2 i}\right) \pi^{S}(\ell)+\beta \omega_{0}^{2} \\
& =\frac{\alpha^{4}}{2 n+1} Q+\frac{\alpha^{2} \gamma^{2}\left(2+\alpha^{2} \gamma^{2}\right)}{2(2 n+1)} \sum_{i=1}^{n} \frac{1}{a_{i}} .
\end{aligned}
$$




$$
\begin{aligned}
G_{\omega^{S}}^{S}\left(x_{0}, x_{2 i}\right) & =G\left(x_{0}, x_{2 i}\right)-\sum_{j=1}^{n}\left[\omega^{S}\left(x_{2 i}\right) G\left(x_{0}, x_{2 j}\right)+\omega^{S}\left(x_{0}\right) G\left(x_{2 i}, x_{2 j}\right)\right] \pi^{S}\left(x_{2 j}\right) \\
& -\omega^{S}\left(x_{2 i}\right) G\left(x_{0}, x_{0}\right) \pi^{S}\left(x_{0}\right)-\omega^{S}\left(x_{0}\right) G\left(x_{2 i}, x_{0}\right) \pi^{S}\left(x_{0}\right)+\beta \omega^{S}\left(x_{0}\right) \omega^{S}\left(x_{2 i}\right) \\
& =\frac{1}{2 n+1}\left(Q-\frac{1}{a_{i}}\right)-\frac{n \alpha^{2} \gamma^{2}}{2(2 n+1)}\left(2 Q-\frac{1}{a_{i}}\right) \\
& -\frac{\alpha^{2} \gamma^{2}}{2(2 n+1)} \sum_{j=1}^{n}\left(Q-\frac{1}{a_{j}}\right)-\frac{\alpha^{2} \gamma^{2}}{2(2 n+1)} \sum_{j=1}^{n}\left(Q-\frac{1}{a_{i}}-\frac{1}{a_{j}}\right)-\frac{\alpha^{2} \gamma^{2}}{2} \frac{1}{a_{i}} \\
& +\left(n^{2} \alpha^{2} \gamma^{4} Q+\frac{\alpha^{2} \gamma^{4}}{2} \sum_{i=1}^{n} \frac{1}{a_{i}}\right) \frac{\alpha^{2}}{2 n+1} \\
& =\frac{\alpha^{4}}{2 n+1} Q+\left(-\frac{1}{2 n+1}+\frac{n \alpha^{2} \gamma^{2}}{2 n+1}-\frac{\alpha^{2} \gamma^{2}}{2}\right) \frac{1}{a_{i}}+\frac{\alpha^{2} \gamma^{2}\left(2+\alpha^{2} \gamma^{2}\right)}{2 n+1} \sum_{j=1}^{n} \frac{1}{a_{j}} \\
& =\frac{\alpha^{4}}{2 n+1} Q-\frac{2+\alpha^{2} \gamma^{2}}{2(2 n+1)} \frac{1}{a_{i}}+\frac{\alpha^{2} \gamma^{2}\left(2+\alpha^{2} \gamma^{2}\right)}{2(2 n+1)} \sum_{j=1}^{n} \frac{1}{a_{j}} .
\end{aligned}
$$

The expression for the Green function for positions involving new vertices are

$$
\begin{aligned}
G_{\omega S}^{S}\left(x_{0}, x_{02 i}\right) & =\frac{1}{2} \gamma \sqrt{2 n+1}\left(G\left(x_{0}, x_{0}\right)+G\left(x_{0}, x_{2 i}\right)\right) \\
& -\left(\frac{\alpha \gamma}{2}\left(G\left(x_{0}, x_{0}\right)+G\left(x_{2 i}, x_{0}\right)\right)+\alpha \gamma G\left(x_{0}, x_{0}\right)\right) \frac{1}{2} n \alpha \gamma^{2} \sqrt{2 n+1} \\
& -\sum_{j=1}^{n}\left(\frac{\alpha \gamma}{2}\left(G\left(x_{0}, x_{2 j}\right)+G\left(x_{2 i}, x_{2 j}\right)\right)+\alpha \gamma G\left(x_{0}, x_{2 j}\right)\right) \frac{1}{2} \alpha \gamma^{2} \sqrt{2 n+1} \\
& +\left(n^{2} \alpha^{4} \gamma^{4} Q+\frac{\alpha^{2} \gamma^{4}}{2} \sum_{j=1}^{n} \frac{1}{a_{j}}-\frac{\gamma^{2}(2 n+1)}{4} \frac{1}{a_{i}}\right) \frac{\alpha^{2} \gamma}{\sqrt{2 n+1}} \\
& =\frac{\alpha^{4} \gamma}{\sqrt{2 n+1}} Q-\frac{\gamma\left(1+(n+1) \alpha^{2} \gamma^{2}\right)}{2 \sqrt{2 n+1}} \frac{1}{a_{i}}+\frac{\alpha^{2} \gamma^{3}\left(2+\alpha^{2} \gamma^{2}\right)}{2 \sqrt{2 n+1}} \sum_{j=1}^{n} \frac{1}{a_{j}} .
\end{aligned}
$$

For $i \neq j$, 


$$
\begin{aligned}
G_{\omega^{S}}^{S}\left(x_{2 i}, x_{02 j}\right) & =\frac{1}{2} \gamma \sqrt{2 n+1}\left(G\left(x_{0}, x_{2 i}\right)+G\left(x_{2 j}, x_{2 i}\right)\right) \\
& -\left(\frac{\alpha \gamma}{2}\left(G\left(x_{0}, x_{0}\right)+G\left(x_{2 j}, x_{0}\right)\right)+\alpha \gamma G\left(x_{2 i}, x_{0}\right)\right) \frac{1}{2} n \alpha \gamma^{2} \sqrt{2 n+1} \\
& -\sum_{k=1}^{n}\left(\frac{\alpha \gamma}{2}\left(G\left(x_{0}, x_{2 k}\right)+G\left(x_{2 j}, x_{2 k}\right)\right)+\alpha \gamma G\left(x_{2 i}, x_{2 j}\right)\right) \frac{1}{2} \alpha \gamma^{2} \sqrt{2 n+1} \\
& +\left(n^{2} \alpha^{4} \gamma^{4} Q+\frac{\alpha^{2} \gamma^{4}}{2} \sum_{k=1}^{n} \frac{1}{a_{k}}-\frac{\gamma^{2}(2 n+1)}{4} \frac{1}{a_{j}}\right) \frac{\alpha^{2} \gamma}{\sqrt{2 n+1}} \\
& =\frac{\alpha^{4} \gamma}{\sqrt{2 n+1}} Q-\frac{\gamma\left(2+\alpha^{2} \gamma^{2}\right)}{2 \sqrt{2 n+1}} \frac{1}{a_{i}}-\frac{\gamma\left(1+(n+1) \alpha^{2} \gamma^{2}\right)}{2 \sqrt{2 n+1}} \frac{1}{a_{j}}+\frac{\alpha^{2} \gamma^{3}\left(2+\alpha^{2} \gamma^{2}\right)}{2 \sqrt{2 n+1}} \sum_{k=1}^{n} \frac{1}{a_{k}}
\end{aligned}
$$

When $i=j$ we get a similar expression

$$
\begin{aligned}
G_{\omega^{S}}^{S}\left(x_{2 i}, x_{02 i}\right) & =\frac{1}{2} \gamma \sqrt{2 n+1}\left(G\left(x_{0}, x_{2 i}\right)+G\left(x_{2 i}, x_{2 i}\right)\right) \\
& -\left(\frac{\alpha \gamma}{2}\left(G\left(x_{0}, x_{0}\right)+G\left(x_{2 i}, x_{0}\right)\right)+\alpha \gamma G\left(x_{2 i}, x_{0}\right)\right) \frac{1}{2} n \alpha \gamma^{2} \sqrt{2 n+1} \\
& -\sum_{k=1}^{n}\left(\frac{\alpha \gamma}{2}\left(G\left(x_{0}, x_{2 k}\right)+G\left(x_{2 i}, x_{2 k}\right)\right)+\alpha \gamma G\left(x_{2 i}, x_{2 k}\right)\right) \frac{1}{2} \alpha \gamma^{2} \sqrt{2 n+1} \\
& +\left(n^{2} \alpha^{4} \gamma^{4} Q+\frac{\alpha^{2} \gamma^{4}}{2} \sum_{k=1}^{n} \frac{1}{a_{k}}-\frac{\gamma^{2}(2 n+1)}{4} \frac{1}{a_{i}}\right) \frac{\alpha^{2} \gamma}{\sqrt{2 n+1}} \\
& =\frac{\gamma}{\sqrt{2 n+1}} Q+\frac{\gamma(n-1)}{\sqrt{2 n+1}} \frac{1}{a_{i}}-\frac{n \gamma^{3} \alpha^{2}}{\sqrt{2 n+1}} Q+\frac{3 n \gamma^{3} \alpha^{2}}{4 \sqrt{2 n+1}} \frac{1}{a_{i}} \\
& -\frac{n \gamma^{3} \alpha^{2}}{\sqrt{2 n+1}} Q+\frac{\gamma^{3} \alpha^{2}}{\sqrt{2 n+1}} \sum_{k=1}^{n} \frac{1}{a_{k}}-\frac{3(n+1) \gamma^{3} \alpha^{2}}{4 \sqrt{2 n+1}} \frac{1}{a_{i}} \\
& +\left(n^{2} \alpha^{4} \gamma^{4} Q+\frac{\alpha^{2} \gamma^{4}}{2} \sum_{k=1}^{n} \frac{1}{a_{k}}-\frac{\gamma^{2}(2 n+1)}{4} \frac{1}{a_{i}}\right) \frac{\alpha^{2} \gamma}{\sqrt{2 n+1}} \\
& =\frac{\alpha^{4} \gamma}{\sqrt{2 n+1}} Q+\frac{\gamma\left(4(n-1)-2(n+2) \alpha^{2} \gamma^{2}\right)}{4 \sqrt{2 n+1}} \frac{1}{a_{i}}+\frac{\alpha^{2} \gamma^{3}\left(2+\alpha^{2} \gamma^{2}\right)}{2 \sqrt{2 n+1}} \sum_{j=1}^{n} \frac{1}{a_{j}} .
\end{aligned}
$$

For positions that involve only new vertices, we get 


$$
\begin{aligned}
& G_{\omega^{S}}^{S}\left(x_{02 i}, x_{02 j}\right)=-\frac{\alpha^{2} \gamma^{4}(2 n+1)}{4}\left(\frac{1}{a_{i}}+\frac{1}{a_{j}}\right)+\beta \alpha^{2} \gamma^{2} \\
& -\frac{n \alpha^{2} \gamma^{4}(2 n+1)}{4}\left(2 G\left(x_{0}, x_{0}\right)+G\left(x_{2 i}, x_{0}\right)+G\left(x_{2 j}, x_{0}\right)\right) \\
& -\frac{\alpha^{2} \gamma^{4}(2 n+1)}{4} \sum_{k=1}^{n}\left(2 G\left(x_{0}, x_{2 k}\right)+G\left(x_{2 i}, x_{2 k}\right)+G\left(x_{2 j}, x_{2 k}\right)\right) \\
& +\frac{\gamma^{2}(2 n+1)}{4}\left(G\left(x_{0}, x_{0}\right)+G\left(x_{0}, x_{2 j}\right)+G\left(x_{2 i}, x_{0}\right)+G\left(x_{2 i}, x_{2 j}\right)\right) \\
& =-\frac{\alpha^{2} \gamma^{4}(2 n+1)}{4}\left(\frac{1}{a_{i}}+\frac{1}{a_{j}}\right)+n^{2} \alpha^{4} \gamma^{6} Q+\frac{\alpha^{4} \gamma^{6}}{2} \sum_{k=1}^{n} \frac{1}{a_{k}} \\
& -\frac{n \alpha^{2} \gamma^{4}}{4}\left(4 Q-\frac{1}{a_{i}}-\frac{1}{a_{i}}\right)-\frac{\alpha^{2} \gamma^{4}}{4} \sum_{k=1}^{n}\left(4 Q-\frac{4}{a_{k}}-\frac{1}{a_{i}}-\frac{1}{a_{j}}\right) \\
& -\frac{\alpha^{2} \gamma^{4}(2 n+1)}{4}\left(\frac{1}{a_{i}}+\frac{1}{a_{j}}\right)+\frac{\gamma^{2}}{4}\left(4 Q-\frac{1}{a_{i}}-\frac{1}{a_{j}}\right) \\
& =\alpha^{4} \gamma^{2} Q-\frac{\gamma^{2}\left(1+(n+1) \alpha^{2} \gamma^{2}\right)}{2}\left(\frac{1}{a_{i}}+\frac{1}{a_{j}}\right)+\frac{\alpha^{2} \gamma^{4}\left(2+\alpha^{2} \gamma^{2}\right)}{2} \sum_{k=1}^{n} \frac{1}{a_{k}} . \\
& G_{\omega^{S}}^{S}\left(x_{02 i}, x_{02 i}\right)=\frac{\gamma^{2}(2 n+1)}{4} \frac{1}{a_{i}}-\frac{\alpha^{2} \gamma^{4}(2 n+1)}{2} \frac{1}{a_{i}}+\beta \alpha^{2} \gamma^{2} \\
& -\frac{n \alpha^{2} \gamma^{4}(2 n+1)}{4}\left(2 G\left(x_{0}, x_{0}\right)+2 G\left(x_{2 i}, x_{0}\right)\right) \\
& -\frac{\alpha^{2} \gamma^{4}(2 n+1)}{4} \sum_{k=1}^{n}\left(2 G\left(x_{0}, x_{2 k}\right)+2 G\left(x_{2 i}, x_{2 k}\right)\right) \\
& +\frac{\gamma^{2}(2 n+1)}{4}\left(G\left(x_{0}, x_{0}\right)+2 G\left(x_{0}, x_{2 i}\right)+G\left(x_{2 i}, x_{2 i}\right)\right) \\
& =\frac{\gamma^{2}(2 n+1)}{4} \frac{1}{a_{i}}-\frac{\alpha^{2} \gamma^{4}(2 n+1)}{2} \frac{1}{a_{i}}+n^{2} \alpha^{4} \gamma^{6} Q+\frac{\alpha^{4} \gamma^{6}}{2} \sum_{k=1}^{n} \frac{1}{a_{k}}-\frac{n \alpha^{2} \gamma^{4}}{4}\left(4 Q-\frac{2}{a_{i}}\right) \\
& -\frac{\alpha^{2} \gamma^{4}}{4} \sum_{k=1}^{n}\left(4 Q-\frac{4}{a_{k}}-\frac{2}{a_{i}}\right)-\frac{\alpha^{2} \gamma^{4}(2 n+1)}{2} \frac{1}{a_{i}}+\frac{\gamma^{2}}{4}\left(4 Q-\frac{4}{a_{i}}\right)+\frac{\gamma^{2}(2 n+1)}{4} \frac{1}{a_{i}} \\
& =\alpha^{4} \gamma^{2} Q-\frac{\gamma^{2}\left(2 n-1-2(n+1) \alpha^{2} \gamma^{2}\right)}{2} \frac{1}{a_{i}}+\frac{\alpha^{2} \gamma^{4}\left(2+\alpha^{2} \gamma^{2}\right)}{2} \sum_{k=1}^{n} \frac{1}{a_{k}} .
\end{aligned}
$$


The remaining cases will follow by performing similar computations.

Finally, we obtain the Kirchhoff index for the partial subdivision of the Star.

Proposition 4.2. Let $S_{2 n}^{S}$ be the partial subdivision network of the star $S_{2 n}$, then the Kirchhoff index of $S_{2 n}^{S}$ is given by

$$
\mathrm{k}_{\omega S}^{S}\left(S_{2 n}^{S}\right)=n\left(2+\alpha^{2} \gamma^{2}\right) Q+\frac{\gamma^{2}}{2}\left(2 n-1-\alpha^{2} \gamma^{2}\right) \sum_{i=1}^{n} \frac{1}{a_{i}} .
$$

Proof. Taking into account Proposition 3.2

$$
\begin{aligned}
\mathrm{k}_{\omega^{S}}^{S}\left(S_{2 n}^{S}\right) & =\mathrm{k}\left(S_{2 n}\right)+\sum_{x \in V} G(x, x) \frac{\pi(x)}{\omega^{S}(x)}-\sum_{x, y \in F} \alpha(x, y) \alpha(y, x) \omega^{S}(x) \omega^{S}(y) R(x, y) \\
& +\sum_{\{x, y\} \in E^{\prime}} \frac{c(x, y)}{c\left(x, v_{x y}\right) c\left(y, v_{x y}\right)}-\beta \\
& =2 n Q+\frac{n \gamma^{2}}{2} Q+\frac{n \gamma^{2}}{2} Q-\gamma^{2} \sum_{i=1}^{n} \frac{1}{a_{i}}+\frac{\gamma^{2}}{2}(2 n+1) \sum_{i=1}^{n} \frac{1}{a_{i}} \\
& -\frac{\gamma^{2}(2 n+1)}{4} \sum_{i=1}^{n} \frac{1}{a_{i}}+\frac{\gamma^{2}(2 n+1)}{4} \sum_{i=1}^{n} \frac{1}{a_{i}}-n^{2} \alpha^{2} \gamma^{4} Q-\frac{\alpha^{2} \gamma^{4}}{2} \sum_{i=1}^{n} \frac{1}{a_{i}} \\
& =\left(2 n+n \gamma^{2}-n^{2} \alpha^{2} \gamma^{4}\right) Q+\frac{\gamma^{2}}{2}\left(2 n-1-\alpha^{2} \gamma^{2}\right) \sum_{i=1}^{n} \frac{1}{a_{i}} .
\end{aligned}
$$

As expected, the Kirchhoff Index of the partial subdivided Star takes the minimum value for $\gamma$ approaching zero; this can be interpreted as no subdivision has been performed in the initial network $S_{2 n}$. Actually, $\mathrm{k}_{\omega S}^{S}\left(S_{2 n}^{S}\right)$ attains a minimum for $\gamma=0$.

\section{Acknowledgments}

This work has been partly supported by the Spanish Research Council (Comisión Interministerial de Ciencia y Tecnología,) under project MTM2017-85996-R.

\section{References}

[1] E. Bendito, A. Carmona and A. M. Encinas, Potential theory for Schrödinger operators on finite networks, Rev. Mat. Iberoamericana 21 (2005) 771-818.

[2] E. Bendito, A. Carmona, A.M. Encinas and J.M. Gesto. Characterization of symmetric $M-$ matrices as resistive inverses. Linear Algebra Appl., 430 (2009) 1336-1349.

[3] T. Biyikoğlu, J. Leydold and P.F. Stadler. Laplacian Eigenvectors of Graphs. LNM 1915, Springer, Berlin, 2007.

[4] C. Bu, B. Yan, X. Zhou, and J. Zhou. Resistance distance in subdivision-vertex join and subdivision-edge join of graphs. Linear Algebra Appl., 458:454-462, 2014.

[5] A. Carmona, A.M. Encinas and M. Mitjana. Discrete elliptic operators and their Green operators. Linear Algebra Appl., 442 (2014) 115-134. 
[6] A. Carmona, M. Mitjana and E. Monsó. Effective resistances and Kirchhoff index in subdivision networks. Linear Multilinear Alg., 65 (2017) 1823-1837.

[7] H. Chen. Random walks and the effective resistance sum rules. Discrete Appl. Math., 158(15):1691-1700, 2010.

[8] D. Cvetković and S. Simić. Graph spectra in Computer science. Linear Algebra Appl., 434 (2011) 1545-1562.

[9] X. Gao, Y. Luo, and W. Liu. Kirchhoff index in line, subdivision and total graphs of a regular graph. Discrete Appl. Math., 160(4-5):560-565, 2012.

[10] A. Ghosh, S. Boyd, and A. Saberi. Minimizing effective resistance of a graph. SIAM Review, 50(1):37-66, 2008.

[11] S. Huang, J. Zhou and C. Bu. Some results on Kirchhoff index and degree-Kirchhoff index. MATCH Commun. Math. Comput. Chem., 75:207-222, 2016.

[12] A.S. Lapaugh and R.L. Rivest. The subgraph homeomorphism problem. J. Comp. Sys. Sciences, 20(2):133-149, 1980.

[13] L. Sun, W. Wang, J. Zhou, and C. Bu. Some results on resistance distances and resistance matrices. Linear Multilinear Algebra, 63(3):523-533, 2015.

[14] W. Xiao and I. Gutman. Resistance distance and Laplacian spectrum. Theor. Chem. Acc., 110(4):284-289, 2003.

[15] Y. Yang. The Kirchhoff index of subdivisions of graphs. Discrete Appl. Math., 171(0):153 $157,2014$.

[16] J. Zhou, L.Sun, and C. Bu Resistance characterization of equiarboreal graphs. Discrete Math., $340: 2864-2870,2017$. 